\title{
Planar Limit of Orientifold Field Theories and Emergent Center Symmetry
}

\author{
Adi Armoni, ${ }^{a}$ Mikhail Shifman, ${ }^{b}$ Mithat $\ddot{\text { Unsal }}{ }^{c, d}$ \\ ${ }^{a}$ Department of Physics, Swansea University, \\ Singleton Park, Swansea, SA2 8PP, UK \\ ${ }^{b}$ William I. Fine Theoretical Physics Institute, \\ University of Minnesota, Minneapolis, MN 55455, USA \\ ${ }^{c}$ SLAC, Stanford University, Menlo Park, CA 94025, USA \\ ${ }^{d}$ Physics Department, Stanford University, Stanford, CA,94305, USA
}

\begin{abstract}
We consider orientifold field theories (i.e. $\mathrm{SU}(N)$ Yang-Mills theories with fermions in the two-index symmetric or antisymmetric representations) on $R_{3} \times S_{1}$ where the compact dimension can be either temporal or spatial. These theories are planar equivalent to supersymmetric Yang-Mills. The latter has $Z_{N}$ center symmetry. The famous Polyakov criterion establishing confinement-deconfinement phase transition as that from $Z_{N}$ symmetric to $Z_{N}$ broken phase applies. At the Lagrangian level the orientifold theories have at most a $Z_{2}$ center. We discuss how the full $Z_{N}$ center symmetry dynamically emerges in the orientifold theories in the limit $N \rightarrow \infty$. In the confining phase the manifestation of this enhancement is the existence of stable $k$-strings in the large- $N$ limit of the orientifold theories. These strings are identical to those of supersymmetric Yang-Mills theories. We argue that critical temperatures (and other features) of the confinement-deconfinement phase transition are the same in the orientifold daughters and their supersymmetric parent up to $1 / N$ corrections. We also discuss the Abelian and non-Abelian confining regimes of four-dimensional QCDlike theories.
\end{abstract}




\section{Introduction}

In this paper we consider dynamical aspects of four-dimensional orientifold field theories compactified on $R_{3} \times S_{1}$. The compactified dimension $S_{1}$ is either temporal or spatial. Whether we deal with thermal or spatial formulation of the problem depends on the spin connection of fermions along the compact direction. In the latter case we arrive at a zero temperature field theory where phase transitions (if any) are induced quantum-mechanically. In either case, if the radius of $S_{1}$ is sufficiently large we return to fourdimensional theory, $R_{3} \times S_{1} \rightarrow R_{4}$.

By orientifold field theories we mean $\mathrm{SU}(N)$ Yang-Mills theories with Dirac fermions in two-index representations of $\mathrm{SU}(N)$ - symmetric or antisymmetric. $^{1}$ Our starting point is the large- $N$ equivalence between these theories and $\mathcal{N}=1$ super-Yang-Mills (SYM) theory $[1,2,3,4,5]$. Since supersymmetric gauge theories are better understood than nonsupersymmetric, we hope to learn more about nonsupersymmetric daughters from planar equivalence. This expectation comes true: the center symmetry of SYM theory turns out to be an emergent symmetry of the orientifold daughters in the large- $N$ limit. This fact was first noted in [6] while the first mention of the problem of center symmetry on both sides of planar equivalence can be found in [7]. Here we investigate the reasons that lead to the emergence of the center symmetry and its implications, as they manifest themselves at small and large values of the $S_{1}$ radius.

For SU(3) the orienti-AS theory reduces to one-flavor QCD. If the large- $N$ limit is applicable to $N=3$, at least semi-quantitatively, we can copy SYM theory data to strongly coupled one-flavor QCD. A concrete example is the temperature independence observed in [8]. It was shown that certain observables of SYM theory are temperature-independent at large $N$ and so is the charge-conjugation-even subset of these observables in the large- $N$ orientifold field theory. It implies a very weak (suppressed by $1 / N$ ) temperature dependence of certain well-defined observables in the confining phase of QCD. This analytical result is supported by lattice simulations $[9,10,11]$. For a recent review, see [12]. The planar equivalence between SYM theory and oreinti-AS is valid in any phase which does not break the charge conjugation symmetry ( $C$ invariance). This implies coinciding Polyakov loop expectation values in

\footnotetext{
${ }^{1}$ They will be referred to as orienti-S and orienti-AS, respectively.
} 
the low-temperature confined and high-temperature deconfined phases and the equality of the the confinement-deconfinement transition temperature in orienti and SYM theories in the large $N$ limit. Other features of the phase transition are predicted to coincide too.

In the spatial compactification of SYM theory, the center symmetry is unbroken at any radius. For orienti-AS, it is dynamically broken, along with $C$ and $C P T$, at small radii, and restored at a critical radius of the order of $\Lambda^{-1}$. These zero-temperature, quantum phase transitions are observed in recent lattice simulations by two independent groups $[13,14]$. The unbroken center symmetry in the small $S^{1}$ regime of the vector-like gauge theories, unlike the dynamically broken center symmetry, leads to Higgsing of the theory. The long-distance dynamics of such QCD-like theories are intimately connected to the Polyakov model [15]. We discuss both the strong coupling and weak coupling confinement regimes. At weak coupling we get Polyakov (Abelian) confinement which is analytically tractable. The region of validity of the Abelian confinement in QCD-like theories is a vanishingly small window which diminishes with increasing $N$. The fact that the Abelian confinement regime is vanishingly small is a consequence of volume independence. We discuss this issue in some detail.

Summarizing, our findings are:

Orientifold field theories on $R_{3} \times S_{1}$ exhibit a number of a priori unexpected features. These theories are planar equivalent to supersymmetric Yang-Mills. The latter has $Z_{N}$ center symmetry. The famous Polyakov criterion establishing confinement-deconfinement phase transition as that from $Z_{N}$ symmetric to $Z_{N}$ broken phase applies. At the Lagrangian level the orientifold theories have at most a $Z_{2}$ center. The full $Z_{N}$ center symmetry dynamically emerges in the orientifold theories in the limit $N \rightarrow \infty$. In the confining phase the manifestation of this enhancement is the existence of stable $k$-strings in the large- $N$ limit of the orientifold theories. These strings are identical to those of supersymmetric Yang-Mills theories. The critical temperatures of the confinement-deconfinement phase transitions are the same in the orientifold daughters and their supersymmetric parent up to $1 / N$ corrections. Depending on the size of $S_{1}$ one can identify the Abelian and non-Abelian confining regimes of four-dimensional QCD-like theories.

The organization of the paper is as follows. In Sect. 2 we outline basic facts on planar equivalence, define the Polyakov line, and spatial Wilson lines. 
Section 3 is devoted to the center symmetry in SYM theory, QCD with fundamental fermions and orienti-S/AS. It gives an alternative derivation of the approximate, exact and emergent center symmetries. In Sect. 4 we discuss the strong-coupling manifestation of the emergent center symmetry: existence and stability of $k$-strings. In Sect. 5 we discuss strong vs. weak coupling regimes. Section 6 is devoted to Polyakov's mechanism of Abelian confinement. In particular, we address the issue how it can be generalized to theories with adjoint and two-index fermions. We confront the thermal confinement-deconfinement phase transitions in SYM and orienti theories in Sect. 7. Here we prove the equality of the critical temperatures at $N \rightarrow$ $\infty$. Finally, Sect. 8 briefly summarizes our results. One-loop potentials are derived in Appendix.

\section{Planar equivalence and Polyakov line}

Planar equivalence is equivalence in the large- $N$ limit of distinct QCD-like theories in their common sectors. Most attention received equivalence between SUSY gluodynamics and its orientifold and $Z_{2}$ orbifold daughters. The Lagrangian of the parent supersymmetric theory is

$$
\mathcal{L}=-\frac{1}{4 g_{P}^{2}} G_{\mu \nu}^{a} G_{\mu \nu}^{a}+\frac{i}{g_{P}^{2}} \lambda^{a \alpha} D_{\alpha \dot{\beta}} \bar{\lambda}^{a \dot{\beta}}
$$

where $\lambda^{a \alpha}$ is the gluino (Weyl) field in the adjoint representation of $\mathrm{SU}(N)$, and $g_{P}^{2}$ stands for the coupling constant in the parent theory. The orientifold daughter is obtained by replacing $\lambda^{a \alpha}$ by the Dirac spinor in the two-index (symmetric or antisymmetric) representation (to be referred to as orienti-S or orienti-AS). The gauge coupling stays intact. To obtain the $Z_{2}$ orbifold daughter we must pass to the gauge group $\mathrm{SU}(N / 2) \times \mathrm{SU}(N / 2)$, replace $\lambda^{a \alpha}$ by a bifundamental Dirac spinor, and rescale the gauge coupling, $g_{D}^{2}=2 g_{P}^{2}$ $[16,17,18,19,20]$. We will focus on orienti-AS. Consideration of orienti-S runs in parallel, with the same conclusions.

Planar equivalence between the parent and daughter theories can be applied in arbitrary geometry, in particular, on $S_{1} \times R_{3}, S_{1} \times S_{3}$ and $R_{4}$. The equivalence implies that correlation functions of the daughter theory are equal to the correlation functions of the parent theory at $N \rightarrow \infty$. Hence, 
in those cases where the underlying "microscopic" symmetries of the planarequivalent partners do not coincide, the theory with a lower microscopic symmetry will reflect the symmetries of the parent theory, which are naively absent in the daughter (or vice versa) [4]. The most profound effect of such a symmetry mismatch — and enhancement — occurs when one dimension is compactified onto a circle, and the symmetry under consideration is the center symmetry. The corresponding order parameter is the Polyakov line which we define below.

Assume that one dimension is compactified (it may be either time or a spatial dimension). For definiteness, we will assume $z$ to be compactified. The Polyakov line (sometimes called the Polyakov loop) is defined as a pathordered holonomy of the Wilson line in the compactified dimension,

$$
\mathcal{U}=P \exp \left\{i \int_{0}^{L} a_{z} d z\right\} \equiv V U V^{\dagger}
$$

where $L$ is the size of the compact dimension while $V$ is a matrix diagonalising $\mathcal{U}$. Moreover,

$$
U=\operatorname{diag}\left\{v_{1}, v_{2}, \ldots, v_{N}\right\} \equiv e^{i a L}
$$

where

$$
a=\sum_{\text {Cartan gen }} a_{c} T^{c} \equiv \operatorname{diag}\left\{a_{1}, a_{2}, \ldots, a_{N}\right\}, \quad \sum_{k=1}^{N} a_{k}=0
$$

It is obvious that

$$
a_{i}=-i \ln v_{i} \bmod 2 \pi .
$$

The planar equivalence implies definite relations among the expectation values of the Polyakov loops in $\mathrm{SU}(N)$ SYM and orienti theories - two gauge theories with distinct center symmetries at the Lagrangian level. In the next section, we will discuss the vacuum structure of these theories from the center symmetry viewpoint. 


\section{Center symmetry (exact and approximate)}

In SYM theory all dynamical fields — gluons and gluinos — are in the adjoint representation of $\mathrm{SU}(N)$. This means that the gauge group is

$$
\mathcal{G}=\mathrm{SU}(N) / Z_{N}
$$

rather than $\mathrm{SU}(N)$. This fact manifests itself as a $Z_{N}$ symmetry on the elementary cell of $\left\{a_{1}, a_{2}, \ldots, a_{N}\right\}$. Under $\operatorname{SU}(N)$ transformations from $Z_{N}$

$$
U \longrightarrow e^{\frac{2 \pi i k}{N}} U, \quad k=0,1, \ldots, N-1 .
$$

The $Z_{N}$ symmetry, usually referred to as the center symmetry, may or may not be spontaneously broken. There is a famous Polyakov criterion regarding confinement/deconfinement transition in $\mathrm{SU}(N)$ Yang-Mills theories. If one considers the Polyakov line along the compactified direction, and its expectation value $\langle\operatorname{Tr} \mathcal{U}\rangle$ does not vanish, the center symmetry is broken implying deconfinement. On the other hand, if $\langle\operatorname{Tr} \mathcal{U}\rangle=0$ the center symmetry is unbroken implying confinement. ${ }^{2}$

Introducing fundamental dynamical fermions removes the center symmetry (see Ref. [21]). However, one can still make sense out of the center symmetry as an approximate symmetry. The simplest way to study the impact of the fundamental fermions is to integrate them out implying the following (formal) result:

$$
\log \operatorname{det}\left(i \gamma_{\mu} D_{\mu}^{\mathrm{F}}-m\right)=\sum_{n \in Z} \sum_{C_{n}} \alpha\left(C_{n}\right) \operatorname{Tr} U\left(C_{n}\right)
$$

where the superscript $\mathrm{F}$ stands for fundamental, $\alpha\left(C_{n}\right)$ are coefficients scaling with $N$ as $O\left(N^{0}\right)$. The integer $n$ is the winding number of the loop $C$ along the $S_{1}$ circle which is valued in the first homotopy group of $S_{1}$,

$$
\pi_{1}\left(S_{1}\right) \sim Z
$$

\footnotetext{
${ }^{2}$ In QCD-like theories with fermions, the boundary conditions on fermions - antiperiodic vs. periodic - (to be denoted as $\left.\mathcal{S}^{\mp}\right)$ determine interpretation of the center symmetry. If the fermions obey $\mathcal{S}^{-}$, the partition function has a thermal interpretation; a change in the (temporal) center symmetry realization must be interpreted in terms of the jump in the free energy of the system. If the fermions obey $\mathcal{S}^{+}$, then the partition function is of the twisted type, $\operatorname{Tr}(-1)^{F} e^{-\beta H}$, and realization of the spatial center symmetry has interpretation in terms of the jump in the vacuum energy.
} 
A small mass $m$ is inserted as an infrared regulator. Note that $\log$ of the determinant in (8), the fermionic contribution to the action expressed in terms of the gluonic observables, scales as $N^{1}$.

The $n=0$ sector has net winding number zero. Hence, the corresponding term in (8) is neutral under the center symmetry transformations. However, for instance, the $n=1$ sector operators are Polyakov loops charged under the center group transformations. Thus, the fermion contribution (8) to the action is explicitly non-invariant with respect to the center symmetry.

A typical term in the sum from the winding class $n$ transforms as

$$
\operatorname{Tr} U\left(C_{n}\right) \rightarrow h^{n} \operatorname{Tr} U\left(C_{n}\right)
$$

where $h \in Z_{N}$. Despite this fact, the center symmetry is an approximate symmetry, since the contribution of the fundamental fermions (8) is suppressed as $1 / N$ relative to the pure glue sector whose action sales as $N^{2}$. At $N=\infty$, the fundamental fermions are completely quenched and the center symmetry becomes exact. The connected correlators or expectation values of the gluonic observables - including the Polyakov loop correlators - are the same as in pure Yang-Mills theory. ${ }^{3}$

For orienti-AS the dynamical AS fermions are not suppressed in the large$N$ limit. The above rationale applicable to fundamental fermions no longer holds. However, planar equivalence will lead us to the same conclusion emergence of an approximate center symmetry at large $N$.

The Lagrangian of the orientifold theories has the form

$$
\mathcal{L}=-\frac{1}{4 g^{2}} G_{\mu \nu}^{a} G_{\mu \nu}^{a}+\frac{i}{g^{2}} \psi_{i j}^{\alpha} D_{\alpha \dot{\beta}} \bar{\psi}^{\dot{\beta} i j}
$$

where $\psi_{i j}$ is the Dirac spinor in the two-index antisymmetric or symmetric representation. Obviously, there is no $Z_{N}$ symmetry at the Lagrangian level. The center symmetry is $Z_{2}$ for even $N$ and none for odd $N$. Indeed, the two-index fermion field, unlike that of gluino, does not stay intact under the action of center elements. The action of a center group element on an adjoint fermion is trivial, $\lambda \rightarrow h \lambda h^{\dagger}=\lambda$. The action on an AS fermion is

\footnotetext{
${ }^{3}$ The above suppression is analogous to the isotopic symmetry in QCD. Since the two lightest flavors are very light compared to the strong scale, $m_{u, d} / \Lambda \ll 1$, QCD possesses an approximate $\mathrm{SU}(2)$ invariance despite the fact that the up and down quark masses differ significantly. The appropriate parameter $m_{u, d} / \Lambda$ plays the same role as $1 / N$.
} 
$\psi \rightarrow h \psi h=h^{2} \psi$. Thus, for $N$ even (odd), $h= \pm 1(h=+1)$ are the center group elements which leave the AS fermion invariant, in accordance with at most a $Z_{2}$ center symmetry for orienti-AS theory.

As was mentioned, the antisymmetric fermions are not suppressed in the large $N$ limit. Integrating out the two-index antisymmetric fermion yields

$$
\begin{aligned}
& \log \operatorname{det}\left(i \gamma_{\mu} D_{\mu}^{\mathrm{AS}}-m\right) \\
& =N^{2} \sum_{n \in Z} \sum_{C_{n}} \frac{\alpha\left(C_{n}\right)}{2}\left\{\left[\frac{\operatorname{Tr}}{N} U\left(C_{n}\right)\right]^{2}-\frac{1}{N} \frac{\operatorname{Tr}}{N} U^{2}\left(C_{n}\right)\right\} .
\end{aligned}
$$

In the large- $N$ limit we can ignore the single-trace terms since they are suppressed by $1 / N$ compared to the $O\left(N^{2}\right)$ double-trace term. The single-trace term contribution scales as that of the fundamental fermions, and is quenched in the same fashion.

A typical double-trace term $\left(\operatorname{Tr} U\left(C_{n}\right)\right)^{2}$ is $O\left(N^{2}\right)$ and is a part of the leading large- $N$ dynamics. Thus, the impact of the two-index antisymmetric fermions on dynamics is as important as that of the glue sector of the theory. The double-trace term is explicitly non-invariant under the $Z_{N}$ center transformations.

We see that in orientifold theories the center symmetry implementation is much less trivial than in theories with fundamental quarks. As was argued in [6], the center symmetry emerges dynamically in the planar limit $N \rightarrow \infty$. Here we will carry out a thorough consideration and present independent albeit related arguments.

The action of the pure glue sector is local and manifestly invariant under the $Z_{N}$ center. Integrating out fermions, induces a nonlocal sum (11) over gluonic observables. This sum includes both topologically trivial loops with no net winding around the compact direction (the $n=0$ term) and nontrivial loops with non-vanishing winding numbers. The topologically trivial loops are singlet under the $Z_{N}$ center symmetry by construction, while the loops with non-vanishing windings are non-invariant.

Let us inspect the $N$ dependence more carefully. If we expand the fermion action in the given gluon background we get

$$
\left\langle\exp \left\{-N^{2} \sum_{n \neq 0} \sum_{C_{n}} \frac{\alpha\left(C_{n}\right)}{2}\left[\frac{\operatorname{Tr}}{N} U\left(C_{n}\right)\right]^{2}\right\}\right\rangle,
$$


where $\langle\ldots\rangle$ means averaging with the exponent combining the gluon Lagrangian with the zero winding number term. This weight function is obviously center-symmetric. If $h$ is an element of the $\mathrm{SU}(N)$ center, a typical term in the sum (12) transforms as

$$
\begin{aligned}
& \left\langle\frac{\operatorname{Tr}}{N} U\left(C_{n}\right) \frac{\operatorname{Tr}}{N} U\left(C_{n}\right)\right\rangle \longrightarrow h^{2 n}\left\langle\frac{\operatorname{Tr}}{N} U\left(C_{n}\right) \frac{\operatorname{Tr}}{N} U\left(C_{n}\right)\right\rangle \\
& =h^{2 n}\left[\left\langle\frac{\operatorname{Tr}}{N} U\left(C_{n}\right)\right\rangle\left\langle\frac{\operatorname{Tr}}{N} U\left(C_{n}\right)\right\rangle+\left\langle\frac{\operatorname{Tr}}{N} U\left(C_{n}\right) \frac{\operatorname{Tr}}{N} U\left(C_{n}\right)\right\rangle_{\text {con }}\right],
\end{aligned}
$$

where we picked up a quadratic term as an example. The connected term in the expression above is suppressed relative to the leading factorized part by $1 / N^{2}$, as follows from the standard $N$ counting, and can be neglected at large $N$. As for the factorized part, planar equivalence implies that all expectation values of multi-winding Polyakov loops are suppressed in the large $N$ limit by $1 / N$,

$$
\begin{aligned}
& \left\langle\frac{1}{N} \operatorname{Tr} U\left(C_{n}\right)\right\rangle^{\mathrm{SYM}}=0, \\
& \left\langle\frac{\operatorname{Tr}}{N} U\left(C_{n}\right)\right\rangle^{\mathrm{AS}}=O\left(\frac{1}{N}\right) \rightarrow 0, \quad n \in Z-\{0\},
\end{aligned}
$$

where the first relation follows from unbroken center symmetry in the SYM theory and the latter is a result of planar equivalence (in the $C$-unbroken, confining phase of orienti-AS).

Consequently, the non-invariance of the expectation value of the action under a global center transformation is

$$
\langle\delta S\rangle=\left\langle S\left(h^{n} \operatorname{Tr} U\left(C_{n}\right)\right)-S\left(\operatorname{Tr} U\left(C_{n}\right)\right)\right\rangle=O\left(\frac{1}{N}\right)\langle S\rangle,
$$

which implies, in turn, dynamical emergence of center symmetry in orientifold theories in the large- $N$ limit. Let us emphasize again that the fermion part of the Lagrangian which explicitly breaks the $Z_{N}$ symmetry is not sub-leading in large $N$. However, the effect of the $Z_{N}$ breaking on physical observables is suppressed at $N \rightarrow \infty$. 
This remarkable phenomenon is a natural (and straightforward) consequence of the large- $N$ equivalence between $\mathcal{N}=1 \mathrm{SYM}$ theory and orientiAS. Despite the fact that the center symmetry in the orienti-AS Lagrangian is at most $Z_{2}$, in the $N=\infty$ limit all observables behave as if they are under the protection of the $Z_{N}$ center symmetry. (This point is also emphasized in [23].) We will refer to this emergent symmetry of the orienti-AS vacuum as the custodial symmetry. The custodial symmetry becomes exact in the $N=\infty$ limit, and is approximate at large $N$.

The immediate implication of this discussion is as follows: when we integrate out fermions in the $N=\infty$ limit, the dynamical pattern in orienti-AS/S in the confined phase simplifies. The sum over all homotopy classes in (11) reduces to a single term - the one over the loops with the vanishing winding number, $\sum_{n \in Z} \alpha\left(C_{n}\right) \rightarrow \alpha\left(C_{0}\right)$. Thus,

$$
\log \operatorname{det}\left(i \gamma_{\mu} D_{\mu}^{\mathrm{AS}}-m\right) \sim N^{2} \sum_{C_{0}} \frac{\alpha\left(C_{0}\right)}{2}\left[\frac{\mathrm{Tr}}{N} U\left(C_{0}\right)\right]^{2}
$$

Consequently, the action and other observables of the $N=\infty$ orienti-AS/S are indistinguishable from the "reduced" theory with action

$$
S^{\text {reduced }}=S^{\mathrm{YM}}+\sum_{C_{0}} \frac{\alpha\left(C_{0}\right)}{2}\left[\frac{\operatorname{Tr}}{N} U\left(C_{0}\right)\right]^{2}
$$

Clearly, the $Z_{N}$ center is a manifest symmetry of the reduced theory. Our derivation also provides a direct derivation of the temperature independence [8] of the orienti-AS theory in the confining low-temperature phase. See also Sect. 7 .

The $Z_{N}$-symmetric vacuum structure at low temperatures, in the $C$ unbroken phase, can also be phrased in terms of the eigenvalue distribution of the Polyakov loop. We want to argue that the vacuum of the orienti-AS theory is invariant under the custodial $Z_{N}$ center symmetry (which is the symmetry of the supersymmetric parent). Let $\rho(\theta)$ denote the distribution of the eigenvalues of the Polyakov loop in orienti-AS in the confined phase. Let us decompose $\rho(\theta)$ into its Fourier modes,

$$
\rho(\theta)=\frac{1}{2 \pi} \sum_{k \in Z} e^{i \theta k} \rho_{k} .
$$


All moments (other than that with $k=0$ ) are restricted to be $O(1 / N)$ due to planar equivalence in the low-temperature phase,

$$
\rho_{k}=\left\langle\frac{\operatorname{Tr}}{N} U^{k}\right\rangle=\frac{1}{N} \sum_{i=1}^{N} e^{i a_{i} k} \rightarrow \int d \theta \rho(\theta) e^{i \theta k} \sim \frac{1}{N}, \quad k \neq 0 .
$$

Consequently, in the $N=\infty$ limit, the eigenvalue distribution of the orientifold theory is flat,

$$
\rho(\theta)=\frac{1}{2 \pi} .
$$

This implies, in particular, that under the action of a center group element, $U \rightarrow U \exp \left\{\frac{2 \pi i k}{N}\right\}$, the eigenvalue distribution

$$
\rho(\theta) \rightarrow \rho\left(\theta+\frac{2 \pi k}{N}\right)=\rho(\theta)
$$

remains invariant.

In line with our conclusion are recent calculations on $S_{3} \times S_{1}[22,23]$ (based on techniques developed in $[24,25]$ ) showing that the vacuum of the large- $N$ orientifold theory in the confining phase is characterized by the distribution (19) i.e. supports the $Z_{N}$ center symmetry rather than the naively expected $Z_{2}$. The authors of Refs. $[23,26]$ also reached the conclusion that perturbative transitions which take place on $S_{3} \times S_{1}$ capture the nature of the nonperturbative transition taking place in the semi-decompactification limit of $R_{3} \times S_{1}$, as probed in lattice simulations.

\section{Manifestation of $Z_{N}$ at strong coupling}

If the circumference of $S_{1}$ is large enough, $L>L_{*}$, we are in the non-Abelian confinement regime both in SYM and orientifold theories. ${ }^{4}$ The signature of the $Z_{N}$ center in SYM theory is the existence of the $k$-strings. The tensions and thicknesses of the $k$-strings are class functions of the center group $Z_{N}$. The planar equivalence implies that the orienti-AS theory must have the very

\footnotetext{
${ }^{4}$ The value of $L_{*}$ will be discussed in Sect. 5 . Since neither parent nor daughter theories have massless states in the limit $L \rightarrow \infty$, where we recover $R_{4}$ geometry, the limit must be smooth.
} 
same $k$-strings despite the presence of the dynamical fermions charged under the center group. Below we discuss how this arises.

The simplest SYM string is a (chromoelectric) flux tube that connects heavy (probe) color sources in the fundamental representation. Usually it is referred to as the fundamental string. The flux tubes attached to color sources in higher representations of $\mathrm{SU}(N)$ are known as $k$-strings, where $k$ denotes the $n$-ality of the color representation under consideration. The $n$-ality of the representation with $\ell$ upper and $m$ lower indices (i.e. $\ell$ fundamental and $m$ antifundamental) is defined as

$$
k=|\ell-m| .
$$

It is clear that for stable strings the maximal value of $k=[N / 2]$ where [ ] denotes the integer part. The stability of these $[N / 2]$ varieties of strings is a question of energetics. For $T_{k} \leq k T_{1}$ which is the observation in lattice studies and certain supersymmetric theories, all $k$ strings are stable [27, 28].

On the other hand, in the orientifold-AS theory at finite $N$ (with $N$ even) the only stable $k$-string is the fundamental one. If $N$ is odd, there are no stable $k$-strings at all, as in QCD with fundamental matter [29]. This is due to the fact that probe charges with even $n$-alities can be completely screened by two-index antisymmetric quarks, while those with odd $n$-alities can be screened down up to a single fundamental index (if $N$ is even) or completely (if $N$ is odd). A similar screening takes place in the orientifold-S theory with the quark $\psi_{[i j]}$ replaced by $\psi_{\{i j\}}$.

However, at $N \rightarrow \infty$ the breaking amplitude of a color singlet into two is $1 / N$ suppressed. Note that the same breaking amplitude in large- $N$ QCD with fundamental fermions dies off as $1 / \sqrt{N}$. Consequently, in the limit $N \rightarrow \infty$ all $k$ strings become stable against breaking, and identical to those of the SYM theory. The $k$-sting tension, in leading order in $N$, is $T_{k}=k T_{1}$, where $T_{1}$ is the tension of the fundamental string, and is marginally stable.

Let $W_{k}(C)$ denote a large Wilson loop in a representation with $n$-ality $k$, with $C$ being the boundary of a surface $\Sigma$. The expectation values of such Wilson operators in orienti-AS with odd $N$ is given by the formula

$$
\left\langle W_{k}(C)\right\rangle_{\mathrm{AS}}=e^{-T_{k} A(\Sigma)}+\frac{1}{N} e^{-\mu_{k} P(C)}
$$

where $T_{k}$ is the string tension, $A(\Sigma)$ denotes the area of the surface spanned by the loop $C$, and $P(C)$ its perimeter (see Sect. 10 of [30]). This formula 
captures two asymptotic regimes and exhibits noncommutativity of the longdistance and large- $N$ limits, a general and quite obvious feature,

$$
\lim _{R \rightarrow \infty} \lim _{N \rightarrow \infty}\left[\frac{\log \left\langle W_{k}(C)\right\rangle_{\mathrm{AS}}}{R}\right] \neq \lim _{N \rightarrow \infty} \lim _{R \rightarrow \infty}\left[\frac{\log \left\langle W_{k}(C)\right\rangle_{\mathrm{AS}}}{R}\right] .
$$

At any finite $N$, at asymptotically large distances, the perimeter law dominates, and the potential asymptotes to a constant, i.e,

$$
\lim _{R \rightarrow \infty} V(R)=\text { const . }
$$

In this regime, infinite separation of probe color charges costs only a finite energy. However, if we take the large- $N$ limit first, linear confinement holds at large distances, i.e,

$$
\lim _{R \rightarrow \infty} V(R)=T_{k} R
$$

Planar equivalence, which implies taking the limit $N \rightarrow \infty$ first, guarantees equality of the string tensions in $N=\infty$ SYM theory and orienti-AS,

$$
T_{k}^{\mathrm{SYM}}=T_{k}^{\mathrm{AS}}, \quad \text { for all } k, \quad N=\infty .
$$

We stress again that, unlike fundamental fermions which are quenched in the large $N$ limit, the two-index antisymmetric (symmetric) fermions are not suppressed. The emergence of $[N / 2]$ varieties of chromoelectric flux tubes is associated with the suppression of quantum fluctuations in the large- $N$ limit. This is a nontrivial dynamical effect.

\section{$5 \quad$ Strong vs. weak coupling}

SYM theory is planar equivalent to the orientifold theories provided $C$ (charge conjugation) invariance is not spontaneously broken $[31,32]$. So far we discussed the large- $L$ limit, i.e. $L \gg \Lambda^{-1}$ where $\Lambda$ is the dynamical scale parameter. In this limit both SYM theory and its non-supersymmetric daughters are expected to confine much in the same way as pure Yang-Mills on $R_{4}$. We will refer to this regime as non-Abelian confinement.

On the other hand, if

$$
L \ll \Lambda^{-1}
$$


the gauge coupling is small at the compactification scale. SYM theory with periodic spin connection (which preserves supersymmetry) undergoes gauge symmetry breaking at the high scale $\sim L^{-1}$. The running law of the fourdimensional gauge coupling is changed at the scale where the gauge coupling is still small, i.e. we are in the weak-coupling Higgs regime. In further descent of the scale the corresponding evolution of the coupling constant is determined by a three-dimensional theory.

In the fully Higgsed regime $a_{i} \neq a_{j}$ for all $i, j=1, \ldots N$. If one chooses a generic set of all different $a_{i}$ 's, SYM theory is maximally Higgsed; more exactly, $\mathrm{SU}(N)$ gauge symmetry is broken down to the maximal Abelian subgroup $\mathrm{U}(1)^{N-1}$. The gauge fields from the Cartan subalgebra (as well as the fermions) remain massless in perturbation theory, ${ }^{5}$ (they will be referred to as "photons") while all other gauge fields acquire masses (they will be referred to " $W$ bosons"). For generic sets of $a_{i}$ there is no regular pattern in the $W$ boson masses. However, if the Higgsed theory is described by $Z_{N}$-symmetric expectation values of the diagonal elements $v_{k}$,

$$
v_{k}=e^{\frac{2 \pi i k}{N}}, \quad k=1, \ldots, N
$$

(or permutations), see Fig. 1, the pattern of the $W$ boson masses is regular.

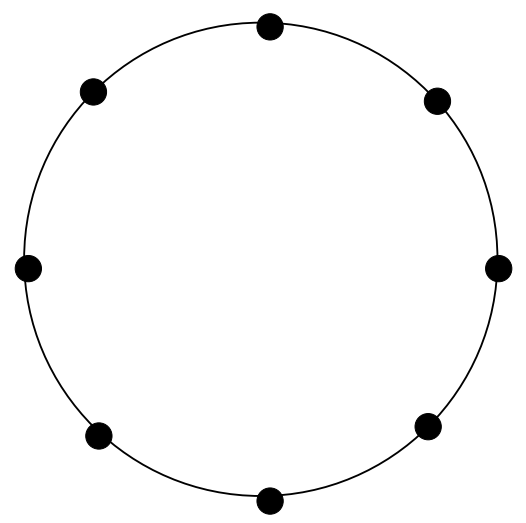

Figure 1: $Z_{N}$ symmetric vacuum fields $v_{k}$. For definitions see Eq. (3).

$N$ lightest $W$ bosons (corresponding to simple roots and affine root of $\mathrm{SU}(N)$ )

\footnotetext{
${ }^{5}$ The nonperturbative mechanism which generates mass gap $\sim \exp \left\{-\frac{8 \pi^{2}}{N g^{2}}\right\}$ is discussed in Sect. 6 .
} 
are degenerate and have masses $\frac{2 \pi}{L N}$, while all others can be obtained as $k \frac{2 \pi}{L N}$ where $k$ is an integer. Thus, there are $\sim N^{2}$ gauge bosons whose mass scales as $1 / L$ and $\sim N$ gauge bosons whose mass scales as $1 /(L N)$.

In the Higgs regime one can consider two distinct sub-regimes. If

$$
L \lesssim L_{*} \equiv \frac{1}{\Lambda N}
$$

there exists a clear-cut separation between the scale of the lightest $W$-bosons $\frac{2 \pi}{L N}$ and nonperturbatively induced photon masses $\sim \exp \left\{-\frac{8 \pi^{2}}{N g^{2}}\right\}$, where $g$ is the gauge coupling in the four-dimensional Lagrangian (1) or (10). How the nonperturbative photon mass is generated and why it leads to linear Abelian confinement is explained in Sect. 6. In this regime, the vast majority of $W$ bosons acquire masses that scale as $N$ and, therefore, decouple in the large$N$ limit. Thus, below the scale $L_{*}^{-1}$ we deal with three-dimensional Abelian theory at weak coupling. In this theory Abelian confinement sets in due to a generalization of the Polyakov mechanism. If the vacuum field is chosen according to (26) the Polyakov order parameter vanishes. We are in the $Z_{N^{-}}$ symmetric regime, much in the same way as in non-Abelian confinement at $L>\Lambda^{-1}$. Note that Eq. (26) implies that

$$
\left\{a_{k} L\right\}=-\frac{2 \pi[N / 2]}{N},-\frac{2 \pi([N / 2]-1)}{N}, \ldots ., \frac{2 \pi[N / 2]}{N},
$$

where $[A]$ stands for the integer part of $A$.

On the other hand, if we increase $L$, starting from $L_{*}$ and eventually approaching $\Lambda^{-1}$, the masses of "typical" $W$ bosons become of order $\Lambda$ (the number of such typical $W$ bosons $\sim N^{2}$, while the masses of "light" $W$ bosons scale as $\Lambda / N$ (the number of light $W$ bosons $\sim N^{1}$ ). In this case the effective low-energy description of the theory at $N \rightarrow \infty$ must include light $W$ 's along with the exponentially light photons. The expectation value of the Polyakov loop still vanishes.

This suggests that the $L$ dependence (in one flavor theories) of all physical quantities is smooth across the board: from $L \lesssim L_{*}$ to $L \gg \Lambda^{-1}$. Rather than a phase transition there may be a crossover. Theoretical considerations at the moment do not allow us to prove or disprove the above conjecture.

The general features of the $L$ behavior are expected to be similar to those of the $\mu$ evolution of the Seiberg-Witten solution of $\mathcal{N}=2$ Yang-Mills 
[33]. (Here $\mu$ is the $\mathcal{N}=2$ breaking parameter in Ref.[33].) If $|\mu|$ is small, the Seiberg-Witten solution applies exhibiting Abelian confinement. As $|\mu|$ evolves to larger values, eventually becoming $\gg \Lambda$, non-Abelian confinement is expected to set in. Since $\mu$ is a holomorphic parameter one does not expect to have a phase transition on the way; the $\mu$ evolution is expected to be smooth.

The nature of the transition between Abelian and non-Abelian confinement a good question for lattice studies. In terms of the expectation value of the Polyakov loop, $\langle\operatorname{Tr} U\rangle=0$ in all regimes. However, at small $L$, this is due to the fact that the eigenvalues of the Polyakov line are, at zeroth order, frozen at the roots of unity as in Fig. 1 (Higgsing), and fluctuations are negligibly small. At large $L$, these eigenvalues are strongly coupled and randomized over the $[0,2 \pi)$ interval. Consequently, there is no gauge symmetry breaking (non-Abelian confinement).

The set of fields in Eq. (28) is automatically invariant under the $C$ transformation. Therefore, planar equivalence between SYM and orientifold theories must hold both in the Higgs regime, and at strong coupling where $C$ invariance was argued to hold too $[32,6]$.

We would like to stress that the set of fields in Eq. (28) should be considered, for the time being, as a fixed background field configuration. Generally speaking, it does not minimize the energy functional. For instance, in the thermal compactification this field configuration realizes the maximum of the effective potential, rather than the minimum. To get the set of fields Eq. (28) as a vacuum configuration (i.e. that minimizing the effective potential) we have to change the pattern of compactification (e.g. $\left.S_{3} \times S_{1}\right)$ or introduce a deformation of the theory through addition of source terms or both. What is important for us here is that this is doable and it is perfectly reasonable to quantize the theory in the $Z_{N}$ invariant background (28) which realizes maximal Higgsing.

Then, it is instructive to illustrate how planar equivalence between SYM and orientifold theories works in this regime by examining the one-loop example. Given the background fields (4) the effective potential for the SYM theory is

$$
V_{\mathrm{eff}}=\frac{1}{24 \pi^{2}}\left\{\sum_{i, j=1}^{N}\left[a_{i}-a_{j}\right]^{2}\left(2 \pi-\left[a_{i}-a_{j}\right]\right)^{2}-\frac{8}{15} \pi^{4} N\right.
$$




$$
\left.-2 \sum_{i<j=1}^{N}\left[a_{i}-a_{j}\right]^{2}\left(2 \pi-\left[a_{i}-a_{j}\right]\right)^{2}\right\},
$$

where everything is measured in the units of $L$. The effective potential for the orientifold-AS theory (see Appendix)

$$
\begin{gathered}
V_{\text {eff }}=\frac{1}{24 \pi^{2}}\left\{\sum_{i, j=1}^{N}\left[a_{i}-a_{j}\right]^{2}\left(2 \pi-\left[a_{i}-a_{j}\right]\right)^{2}-\frac{8}{15} \pi^{4} N\right. \\
\left.-2 \sum_{i<j=1}^{N}\left[a_{i}+a_{j}\right]^{2}\left(2 \pi-\left[a_{i}+a_{j}\right]\right)^{2}\right\},
\end{gathered}
$$

For the $Z_{N}$ symmetric background (4) the expressions (29) and (30) are identical up to terms suppressed by powers of $1 / N$.

\section{Polyakov's 3D confinement}

Long ago Polyakov considered three-dimensional SU(2) Georgi-Glashow model (a Yang-Mills adjoint Higgs system) in the Higgs regime [15]. In this regime $\mathrm{SU}(2)$ is broken down to $\mathrm{U}(1)$, so that at low energies the theory

reduces to compact electrodynamics. The dual photon is a scalar field $\sigma$ of the phase type (i.e. it is defined on the interval $[0,2 \pi]$ ):

$$
F_{\mu \nu}=\frac{g_{3}^{2}}{4 \pi} \varepsilon_{\mu \nu \rho}\left(\partial^{\rho} \sigma\right)
$$

where $g_{3}^{2}$ is the three-dimensional gauge coupling with mass dimension $\left[g_{3}^{2}\right]=$ +1 . In perturbation theory the dual photon $\sigma$ is massless. However, it acquires a mass due to instantons (technically, the latter are identical to the 't Hooft-Polyakov monopoles, after the substitution of one spatial dimension by imaginary time). In the vacuum of the theory, one deals with a gas of instantons interacting according to the Coulomb law. The dual photon mass is due to the Debye screening. In fact, it is determined by the one-instanton vertex,

$$
m_{\sigma} \sim m_{W}^{5 / 2} g_{3}^{-3} e^{-S_{0} / 2}
$$


where $S_{0}$ is the one-instanton action,

$$
S_{0}=4 \pi \frac{m_{W}}{g_{3}^{2}}
$$

$m_{W}$ is the $W$ boson mass. As a result, the low-energy theory is described by a three-dimensional sine-Gordon model,

$$
\mathcal{L}_{\sigma}=\frac{g_{3}^{2}}{32 \pi^{2}}\left(\partial_{\mu} \sigma\right)^{2}+c_{1} m_{W}^{5} g_{3}^{-4} e^{-S_{0}} \cos \sigma
$$

where $c_{1}$ is an undetermined prefactor. This model supports a domain line ${ }^{6}$ (with $\sigma$ field vortices at the endpoints) which in $1+2$ dimensions must be interpreted as a string. Since the $\sigma$ field dualizes three-dimensional photon, the $\sigma$ field vortices in fact represent electric probe charges in the original formulation, connected by the electric flux tubes which look like domain lines in the dual formulation.

As well-known [34], addition of one (or more) Dirac fermions in the adjoint representation eliminates the above Abelian confinement in the Polyakov model. This is due to the fact that the instanton-monopole acquires fermion zero modes due to the Callias index theorem [35, 36]. Instanton-monopoles, instead of generating mass for the the $\sigma$ field through the potential $e^{i \sigma}+e^{-i \sigma}$, produce a vertex with compulsory fermion zero modes attached to it. For one flavor theory, this is given by

$$
e^{-S_{0}}\left(e^{i \sigma} \psi \psi+e^{-i \sigma} \bar{\psi} \bar{\psi}\right)
$$

The three-dimensional microscopic theory with an adjoint fermion possess a non-anomalous $\mathrm{U}(1)$ fermion number symmetry. This $\mathrm{U}(1)$ invariance is manifest in Eq. (35); it intertwines the fermion global rotation with a continuous shift symmetry for the dual photon,

$$
\psi \rightarrow e^{i \alpha} \psi, \quad \sigma \rightarrow \sigma-2 \alpha
$$

The continuous shift symmetry (unlike a discrete one) prohibits any mass term (or potential) for the $\sigma$ field. As was shown in [34], the U(1) fermion number is spontaneously broken, and the dual photon $\sigma$ is the associated Goldstone particle. Thus, the $\sigma$ field remains massless nonperturbatively,

\footnotetext{
${ }^{6}$ Similar to the axion domain wall.
} 
and linear confinement does not occur. This is because domain lines become infinitely thick (infinite range) in the absence of the dual photon mass. One does need a non-vanishing dual photon mass to make the domain line thickness of the order of $m_{\sigma}^{-1}$. Only then, at distances $\gg m_{\sigma}^{-1}$ linear confinement will set in.

As was noted in [37], this obstacle is circumvented if we consider a three-dimensional model obtained as a low-energy reduction of the fourdimensional model compactified on $S_{1} \times R_{3}$. The adjoint Weyl fermion in four dimensions becomes an adjoint Dirac fermion in three dimensions. In this case, there is no $\mathrm{U}(1)$ fermion number symmetry. There is an anomalous $\mathrm{U}(1)_{A}$; because of the anomaly only a discrete subgroup of $\mathrm{U}(1)_{A}$ is a valid symmetry. For SYM, the anomaly free subgroup is $Z_{2 N, A}$. As stated earlier, the discrete shift symmetry does not prohibit a mass term for the dual photon, hence it must be generated [37]. Whatever non-perturbative object is responsible for the dual photon mass, it should have no fermionic zero mode "attached." Otherwise, it will generate vertices as in (35) which do not result in the bosonic potential.

The microscopic origin of the mass term can be traced to the compactness of the adjoint Higgs field whenever we consider a theory on $S_{1} \times R_{3}$. This is the feature which is absent in the Polyakov model [15] and its naive fermionic extension [34]. When the adjoint Higgs field is compact as in Fig.1, in additional to $N-1$ 't Hooft-Polyakov monopole-instantons (whose existence is tied up to $\left.\pi_{1}\left(S_{1}\right) \neq 0\right)$ there is one extra, which can be referred to as the Kaluza-Klein (KK) monopole-instanton. ${ }^{7}$ Each of these monopoles carries two zero modes, hence they cannot contribute to the bosonic potential. The bound state of the 't Hooft-Polyakov monopole-instanton with

\footnotetext{
${ }^{7}$ The eigenvalues shown in Fig. 1 may be viewed as Euclidean D2-branes. $N$ split branes support a spontaneously broken $\mathrm{U}(1)^{N}$ gauge theory, whose $\mathrm{U}(1)$ center of mass decouples, and the resulting theory is $\mathrm{U}(1)^{N-1}$. The $N-1$ BPS monopoles may be viewed as the Euclidean D0 branes connecting eigenvalues $\left(a_{1} \rightarrow a_{2}\right),\left(a_{2} \rightarrow a_{3}\right), \ldots,\left(a_{N-1} \rightarrow a_{N}\right)$. Clearly, we can also have a monopole which connects $\left(a_{N} \rightarrow a_{1}\right)$ which owes its existence to the periodicity of the adjoint Higgs field, or equivalently, to the fact that the underlying theory is on $S_{1} \times R_{3}$. Usually it is called the KK monopole. The Euclidean D0 branes with the opposite orientation, connecting $\left(a_{j} \leftarrow a_{j+1}\right), j=1, \ldots N$ are the antimonopoles. This viewpoint makes manifest the fact that the KK and 't Hooft-Polyakov monopoles are all on the same footing. The magnetic and topological charges of the monopoles connecting $\left(a_{j} \leftrightarrow a_{j+1}\right)$ is $\pm\left(\boldsymbol{\alpha}_{j}, \frac{1}{N}\right)$ where the direction of the arrow is correlated with the sign of the charges.
} 
magnetic charge $\boldsymbol{\alpha}_{i}$ and anti-monopole with charge $-\boldsymbol{\alpha}_{i+1}$ has no fermion zero mode: in the sense of topological charge, it is indistinguishable from the perturbative vacuum. Hence, such a bound state can contribute to the bosonic potential. If we normalize the magnetic and topological charges of the monopoles as

$$
\left(\int F, \int F \tilde{F}\right)=\left(\boldsymbol{\alpha}_{i}, \frac{1}{N}\right),
$$

where $\boldsymbol{\alpha}_{i}$ stand for roots of the affine Lie algebra, then the following bound states are relevant:

$$
\left(\boldsymbol{\alpha}_{i}, \frac{1}{N}\right)+\left(-\boldsymbol{\alpha}_{i+1},-\frac{1}{N}\right)=\left(\boldsymbol{\alpha}_{i}-\boldsymbol{\alpha}_{i+1}, 0\right) .
$$

This pair is stable, as was shown in Ref. [37], where it is referred as a magnetic bion. Thus, we can borrow Polyakov's discussion of magnetic monopoles and apply directly to these objects. The magnetic bions will induce a mass term for the dual photons via the Debye screening, the essence Polyakov's mechanism.

In the $\mathrm{SU}(N)$ gauge theory on $R_{3} \times S_{1}$, which is Higgsed, $\mathrm{SU}(N) \rightarrow$ $U(1)^{N-1}$, the bosonic part of the effective low-energy Lagrangian is generated by the pairs (38), and hence the potential is proportional to $e^{-2 S_{0}}$, rather than $e^{-S_{0}}$ in the Polyakov problem. If we introduce an $(N-1)$-component vector $\sigma$

$$
\boldsymbol{\sigma} \equiv\left(\sigma_{1}, \ldots, \sigma_{N-1}\right),
$$

representing $N-1$ dual photons of the $U(1)^{N-1}$ theory, and $\boldsymbol{\alpha}_{i}(i=1, \ldots, N)$ are the simple and affine roots of the $\mathrm{SU}(N)$ Lie algebra, the bosonic part of the effective Lagrangian can be written as

$$
\mathcal{L}\left(\sigma_{1}, \ldots, \sigma_{N-1}\right)=\frac{g_{3}^{2}}{32 \pi^{2}}\left(\partial_{\mu} \boldsymbol{\sigma}\right)^{2}+c m_{W}^{5} g_{3}^{-4} e^{-2 S_{0}} \sum_{i=1}^{N} \cos \left(\boldsymbol{\alpha}_{i}-\boldsymbol{\alpha}_{i+1}\right) \boldsymbol{\sigma},
$$

where $c$ is an undetermined coefficient and $g_{3}$ is the three-dimensional coupling constant,

$$
g_{3}^{2}=g^{2} L^{-1}
$$

We remind that $\boldsymbol{\alpha}_{i}(i=1, \ldots, N-1)$ represent the magnetic charges of $(N-1)$ types of the 't Hooft-Polyakov monopoles while the affine root

$$
\boldsymbol{\alpha}_{N}=-\sum_{i=1}^{N-1} \boldsymbol{\alpha}_{i}
$$


is the magnetic charge of the KK monopole. Note that the configurations that contribute to the effective Lagrangian have magnetic charges $\boldsymbol{\alpha}_{i}-\boldsymbol{\alpha}_{i+1}$ and vertices $e^{i\left(\boldsymbol{\alpha}_{i}-\boldsymbol{\alpha}_{i+1}\right) \boldsymbol{\sigma}}$, corresponding to a product of a monopole vertex $e^{i \boldsymbol{\alpha}_{i} \boldsymbol{\sigma}}$ with charge $\boldsymbol{\alpha}_{i}$, and antimonopole vertex $e^{-i \boldsymbol{\alpha}_{i+1} \boldsymbol{\sigma}}$ with charge $-\boldsymbol{\alpha}_{i+1}$. We used Eq. (28) to guarantee that the vacuum configuration is $Z_{N}$-symmetric, hence the actions (fugacities) $e^{-2 S_{0}}$ are all equal.

Equation (40) implies that non-vanishing masses are generated for all $\sigma$, proportional to $e^{-S_{0}}$, albeit much smaller than the masses in the Polyakov model in which they are $\sim e^{-S_{0} / 2}$. There are $N-1$ distinct $\mathrm{U}(1)$ 's in this model, corresponding to $N-1$ distinct electric charges. These are the electric charges $q_{i}$ of all color components of a probe non-dynamical quark $Q_{i}$ $(i=1, \ldots, N)$ in the fundamental representation of $\mathrm{SU}(N) .{ }^{8}$ Correspondingly, there are $N-1$ types of Abelian strings (domain lines). Their tensions are equal to each other and proportional to $e^{-S_{0}}$. Linear confinement develops at distances larger than $e^{S_{0}}$.

Needless to say, the physical spectrum in the Higgs/Abelian confinement regime is much richer than that in the non-Abelian confinement regime. If in the latter case only color-singlets act as asymptotic states, in the Abelian confinement regime all systems that have vanishing $N-1$ electric charges have finite mass and represent asymptotic states.

\section{Thermal compactification}

As was already mentioned the requirement for planar equivalence to hold is to have a vacuum with an unbroken $C$ conjugation symmetry. In the non-Abelian confinement regime $C$ parity is unbroken. In the Higgs regime this depends on the choice of $v_{k}$ 's. The set (26) automatically guarantees $C$ parity. Now we abandon this choice and will focus on the case $a_{k}=0, \pi$ relevant to the thermal compactification. The vacuum field $a_{k}=0$ or $a_{k}=\pi$ for all $k$ is not $Z_{N}$ symmetric while, as we already know, the non-Abelian confinement regime is $Z_{N}$-symmetric. Hence, we expect a phase transition at a deconfinement temperature $T_{*}$.

When the temperature is high, namely $T>T_{*}$ (the deconfining phase), the Polyakov loop expectation value in orienti-AS/S does not vanish; the

\footnotetext{
${ }^{8} q_{i}$ are subject to the condition $\sum_{i=1}^{N} q_{i}=0$.
} 
minimal energy states are $C$ preserving vacua $a_{k}=0$ or $a_{k}=\pi$ for all $k$. If we choose the same minima of the effective potential in the high-temperature phase of the SYM theory, planar equivalence will hold. Namely, the SYM theory and orienti-AS/S will have the same Green functions, condensates, spectra, etc. in the common sector.

Since all the eigenvalues of the Polyakov line coincide in the high temperature phase, say at $a_{k}=0$ for all $k$, the high-temperature theory is not Higgsed. The would-be $W$ bosons remain massless. Its dynamics is that of the non-Abelian theory, albeit three-dimensional rather than four-dimensional. It is believed that the phase transition at $T_{*}$ separates the $Z_{N}$ symmetric phase from that with broken $Z_{N}$ in pure Yang-Mills or SYM theory. The orientifold theories seemed puzzling from this standpoint [7]. Now we understand that the phase transition at $T_{*}$ in orienti-AS/S is quite similar: it separates the high-temperature phase with no $Z_{N}$ center symmetry (at best, it is $Z_{2}$ for even $N$ which is spontaneously broken) from the low-temperature phase with emergent unbroken $Z_{N}$.

The physical observables in the common sector of SYM theory and orientiAS/S must coincide throughout the whole phase diagram, since charge conjugation symmetry is unbroken in neither phase of the theory. This implies, in particular, the phase transition temperatures must coincide,

$$
T_{*}^{\mathrm{SYM}}=T_{*}^{\text {orienti-AS/S }}, \quad N \rightarrow \infty .
$$

The same applies to the Polyakov order parameter. At $N=\infty$,

$$
\left\langle\frac{1}{N} \operatorname{Tr} U\right\rangle^{\mathrm{SYM}, \text { orienti-AS/S }}= \begin{cases}0, & T<T_{*} \\ \pm 1, & T>T_{*}\end{cases}
$$

where the sign double-valuedness corresponds to two possible vacuum fields, $a_{k}=0, \forall k$ or $a_{k}=\pi, \forall k$. (In SYM theory these two minima correspond to the ones invariant under the naive $C$, where the comparison is straightforward.) In other words, the notions of high and low temperatures, defined relatively the deconfinement transition temperature must coincide in the two theories. Otherwise we would get an inconsistency among the common sector observables. This gives us a nontrivial dynamical result, at finite $N$,

$$
T_{*}^{\mathrm{SYM}}=T_{*}^{\text {orienti-AS/S }}\left[1+O\left(\frac{1}{N}\right)\right] .
$$

This prediction of planar equivalence should be easily testable on lattices. 


\section{Conclusions}

The strong coupling dynamics of non-supersymmetric vector-like gauge theories, despite many efforts over the years, remains elusive. Currently, the nonperturbative large- $N$ equivalences provide deep hints into the structure of the vector-like gauge theories. One of the most profound examples of the large- $N$ equivalences is that between $\mathcal{N}=1 \mathrm{SYM}$ theory and its orientifold daughters. The parent and daughter theories are clearly distinct, with different fundamental symmetries and dynamics. At the Lagrangian level, SYM theory is supersymmetric and has $Z_{N}$ center symmetry, while orienti theories are non-supersymmetric and have at most a $Z_{2}$ center.

However, the large- $N$ equivalence tells us that these theories become indistinguishable in their neutral sector in the large- $N$ limit. More specifically, it tells us that the physical Hilbert spaces of these two theories in the $C$ even subsectors coincide, their confinement-deconfinement temperatures are identical, and the $k$-string tensions must match.

Apparently, all these observables are associated with certain symmetries which are explicit in the parent theory, but not in the daughter ones. In the large- $N$ limit the correlators of the daughter theories carry benchmarks of the custodial symmetries of its parent. We refer to such symmetries, which are absent at the Lagrangian level but appear dynamically in the neutral correlators, as to emergent symmetries. In orienti-AS, the $Z_{N}$ center symmetry and supersymmetry (protecting the degeneracy of the bosonic spectrum) are emergent symmetries in this sense.

At the level of the Schwinger-Dyson equations (or loop equations), the equivalence is a consequence of the quantum fluctuation suppression in the large- $N$ limit. The (suppressed) fluctuations are well aware of distinctions in the parent/daughter theories, which have no place in the leading large- $N$ dynamics. This is true for orienti-AS/S, as well as for orbifold $\mathrm{SU}(N) \times \mathrm{SU}(N)$ Yang-Mills with bifundamental quark (assuming unbroken $Z_{2}$ interchange symmetry). This is also valid for-one flavor QCD with orthogonal and symplectic gauge groups with AS/S representations fermions [39]. In our opinion, attempts to understand such universal behavior (which is a natural consequence of planar equivalence) may provides insights into the strongly coupled regimes of QCD, and other strongly coupled system. This question is left for the future work. 


\section{Appendix: One-loop potentials}

The one-loop effective potentials for Polyakov lines in the case of SYM theory and orienti-AS are

$$
\begin{aligned}
& V_{\mathrm{eff}}^{\mathrm{SYM}}[U]=\frac{2}{\pi^{2} L^{4}} \sum_{n=1}^{\infty} \frac{1}{n^{4}}\left[\left(-1+a_{n}\right)\left|\operatorname{Tr} U^{n}\right|^{2}\right] \\
& V_{\mathrm{eff}}^{\mathrm{A}}[U]=\frac{2}{\pi^{2} L^{4}} \sum_{n=1}^{\infty} \frac{1}{n^{4}}\left\{-\left|\operatorname{Tr} U^{n}\right|^{2}+a_{n}\left[\frac{\left(\operatorname{Tr} U^{n}\right)^{2}-\operatorname{Tr} U^{2 n}}{2}+\text { c.c. }\right]\right\}
\end{aligned}
$$

where the first terms are due to gauge boson (and ghosts), and second term is due to fermions endowed with spin structure

$$
a_{n}=\left\{\begin{array}{cc}
(-1)^{n} & \text { for } \mathcal{S}^{-} \\
1 & \text { for } \mathcal{S}^{+}
\end{array}\right.
$$

In the large $N$ limit, the single trace term can be neglected since it is suppressed by $O(1 / N)$ relative to the double trace terms. In terms of the simultaneous eigenstates of the $C$ and center symmetry,

$$
\operatorname{Tr} \Omega_{ \pm}^{n}=\operatorname{Tr} U^{n} \pm \operatorname{Tr}\left(U^{*}\right)^{n}, \quad C \operatorname{Tr} \Omega_{ \pm}= \pm \operatorname{Tr} \Omega_{ \pm}
$$

the potential takes the form

$$
V_{\mathrm{eff}}^{\mathrm{AS}}\left[\Omega_{+}, \Omega_{-}\right]=\frac{2}{\pi^{2} L^{4}} \sum_{n=1}^{\infty} \frac{1}{4 n^{4}}\left[\left(-1+a_{n}\right)\left|\operatorname{Tr} \Omega_{+}^{n}\right|^{2}+\left(-1-a_{n}\right)\left|\operatorname{Tr} \Omega_{-}^{n}\right|^{2}\right] .
$$

The form of the one-loop potential in $\mathrm{QCD}(\mathrm{AS})$ is not surprising. The first class of terms $\left|\operatorname{Tr} \Omega_{+}^{n}\right|^{2}$ are the images of the $|\operatorname{Tr} U|^{2}$. The second category are the square of the twisted (non-neutral) $C$-odd operators, which are not the image of any operator in the orienti-partner. They do, however, get induced via a one loop Coleman-Weinberg analysis. Whether or not the twisted operator $\operatorname{Tr} \Omega_{-}$acquires a vacuum expectation value and induces the spontaneous braking of charge conjugation is correlated with the spin structure $\mathcal{S}^{ \pm}$ of fermions along the $S_{1}$ circle. In thermal case $\left(\mathcal{S}^{+}\right)$, despite the presence 
of such operators, $C$ is unbroken at high temperature. In spatial compactification $\left(\mathcal{S}^{-}\right), C$ is broken at small $S_{1}$. Regardless of spin structure, $C$ is preserved at large radius (either temporal or spatial) continuously connected to $R_{4}$.

\section{Acknowledgments}

We are grateful to Larry Yaffe for discussions. A.A. is supported by the PPARC advanced fellowship award. The work of M.S. is supported in part by DOE grant DE-FG02-94ER408. The work of M.Ü. is supported by the U.S. Department of Energy Grants DE-AC02-76SF00515.

\section{References}

[1] A. Armoni, M. Shifman and G. Veneziano, Nucl. Phys. B 667, 170 (2003) [arXiv:hep-th/0302163]; Phys. Rev. Lett. 91, 191601 (2003) [arXiv:hep-th/0307097]; Phys. Lett. B 579, 384 (2004) [arXiv:hepth/0309013].

[2] A. Armoni, M. Shifman and G. Veneziano, From super-Yang-Mills theory to QCD: Planar equivalence and its implications, in From Fields to Strings: Circumnavigating Theoretical Physics, Ed. M. Shifman, A. Vainshtein, and J. Wheater (World Scientific, Singapore, 2005), Vol. 1, p. 353, [arXiv:hep-th/0403071].

[3] A. Armoni, M. Shifman and G. Veneziano, Phys. Rev. D 71, 045015 (2005) [arXiv:hep-th/0412203].

[4] P. Kovtun, M. Ünsal, and L. G. Yaffe, JHEP 0312, 034 (2003) [arXiv:hep-th/0311098].

[5] P. Kovtun, M. Ünsal, and L. G. Yaffe, JHEP 0507, 008 (2005) [arXiv:hep-th/0411177].

[6] M. Shifman, Some Theoretical Developments in SUSY, arXiv:0708.0632 [hep-th]. 
[7] F. Sannino, Phys. Rev. D 72, 125006 (2005) [arXiv:hep-th/0507251].

[8] P. Kovtun, M. Ünsal, and L. G. Yaffe, JHEP 0706, 019 (2007) [arXiv:hep-th/0702021].

[9] B. Lucini, M. Teper and U. Wenger, JHEP 0502, 033 (2005) [arXiv:heplat/0502003].

[10] B. Lucini, M. Teper and U. Wenger, JHEP 0406, 012 (2004) [arXiv:heplat/0404008].

[11] R. Narayanan and H. Neuberger, Phys. Rev. Lett. 91, 081601 (2003) [arXiv:hep-lat/0303023].

[12] R. Narayanan and H. Neuberger, arXiv:0710.0098 [hep-lat].

[13] T. DeGrand and R. Hoffmann, JHEP 0702, 022 (2007) [arXiv:heplat/0612012].

[14] B. Lucini, A. Patella and C. Pica, Phys. Rev. D 75, 121701 (2007) [arXiv:hep-th/0702167].

[15] A. M. Polyakov, Nucl. Phys. B 120, 429 (1977).

[16] A. Armoni and B. Kol, JHEP 9907, 011 (1999) [arXiv:hep-th/9906081].

[17] M. J. Strassler, On methods for extracting exact non-perturbative results in non-supersymmetric gauge theories, arXiv:hep-th/0104032.

[18] D. Tong, JHEP 0303, 022 (2003) [arXiv:hep-th/0212235].

[19] A. Armoni, A. Gorsky and M. Shifman, Phys. Rev. D 72, 105001 (2005) [arXiv:hep-th/0505022].

[20] P. Kovtun, M. Ünsal and L. G. Yaffe, Phys. Rev. D 72, 105006 (2005) [arXiv:hep-th/0505075].

[21] N. Weiss, Phys. Rev. D 24, 475 (1981).

[22] T. J. Hollowood and A. Naqvi, JHEP 0704, 087 (2007) [arXiv:hepth/0609203]; see also [23]. 
[23] M. Ünsal, Phys. Rev. D 76, 025015 (2007) [arXiv:hep-th/0703025].

[24] B. Sundborg, Nucl. Phys. B 573, 349 (2000) [arXiv:hep-th/9908001].

[25] O. Aharony, J. Marsano, S. Minwalla, K. Papadodimas and M. Van Raamsdonk, Adv. Theor. Math. Phys. 8, 603 (2004) [arXiv:hepth/0310285].

[26] C. Hoyos-Badajoz, B. Lucini and A. Naqvi, arXiv:0711.0659 [hep-th].

[27] B. Lucini and M. Teper, Phys. Rev. D 64, 105019 (2001) [arXiv:heplat/0107007].

[28] A. Armoni and B. Lucini, JHEP 0606, 036 (2006) [arXiv:hepth/0604055].

[29] S. Kratochvila and P. de Forcrand, Nucl. Phys. B 671, 103 (2003) [arXiv:hep-lat/0306011].

[30] A. Armoni and M. Shifman, Nucl. Phys. B 671, 67 (2003) [arXiv:hepth/0307020].

[31] M. Unsal and L. G. Yaffe, Phys. Rev. D 74, 105019 (2006) [arXiv:hepth/0608180].

[32] A. Armoni, M. Shifman and G. Veneziano, Phys. Lett. B 647, 515 (2007) [arXiv:hep-th/0701229].

[33] N. Seiberg and E. Witten, Nucl. Phys. B 426, 19 (1994) [Erratum-ibid. B 430, 485 (1994)] [arXiv:hep-th/9407087].

[34] I. Affleck, J. A. Harvey and E. Witten, Nucl. Phys. B 206, 413 (1982).

[35] C. Callias, Commun. Math. Phys. 62, 213 (1978).

[36] R. Jackiw and C. Rebbi, Phys. Rev. D 13, 3398 (1976).

[37] M. Ünsal, Abelian duality, confinement, and chiral symmetry breaking in $Q C D$ (adj), arXiv:0708.1772 [hep-th].

[38] M. Ünsal, Magnetic bion condensation: A new mechanism of confinement and mass gap in four dimensions, arXiv:0709.3269 [hep-th]. 
[39] A. Armoni, JHEP 0704, 046 (2007) [arXiv:hep-th/0703229]. 